\title{
A IMPORTÂNCIA DO DIAGNÓSTICO EM NÍVEL LOCAL PARA O PLANEJAMENTO DE SERVIÇOS DE SAÚDE MATERNA
}

\author{
Sueli Gandolfi GIRON * \\ Wanda MARKIEVICZ ** \\ Maria Aparecida NOVAES ** \\ Neia SCHOR * \\ Cyro CIARI JR. * \\ Arnaldo A. F. de SIQUEIRA*
}

RSPU-B / 202

Grron, S. G. et al. - A importancia do diagnóstico em nível local para o planejamento de servico de saide materna. Rev. Saúde públ., S. Paulo, 8: $43-9,1974$.

Resumo: Foi analisada uma área do municipio de Osasco no Estado de Säo Paulo, com 40.134 habitantes para verificar o grau de atendimento em saude materna, recebido pelas gestantes dessa área, que não contava com recursos oficiais de pré-natal. Foi construido um formulário aplicado a uma amostra de 1.036 residências. Os resultados evidenciaram um numero elevado de partos hospitalares, alta concentração de consultas de pré-natal por gestantes (6,27), e que estas eram atendidas por sociedades de medicina de grupo. Concluiu-se que do se programar servicos de saúde materna para uma área é imperativo um diagnóstico prévio das necessidades e recomenda-se que os órgãos oficiais de Saúde Pública considerem todos os recursos de saúde da área e os assessore quando existentes.

UNITERMS: Saúde materna*; Assistência pré-natal*; Gravidez*; Saúde publica.

I N T R O D U C A O

A Secretaria de Saúde do Estado de São Paulo ao definir os programas que deveriam ser desenvolvidos nos diversos tipos de unidade sanitária de sua rede, houve por bem colocar programas de saúde materna em todos os níveis. Esse fato é amplamente justificado teoricamente, devido aos altos índices de mortalidade e morbidade que afetam o grupo gestante no Brasil, e em São Paulo, especifica- mente ${ }^{2}$. Sabe-se, também, que as condiçôes de saúde do recém-nascido dependem do grau de normalidade do período gestacional, podendo-se atribuir parte da da mortalidade e morbidade da fase perinatal àquele período. A Investigação Interamericana de Mortalidade na Infância ${ }^{4}$ mostrou que o número de óbitos de crianças menores de cinco anos é inversamente proporcional à atenção pré-

* Do Departamento de Prática de Saúde Pública da Faculdade de Saúde Pública da USP - Av. Dr. Arnaldo, 715 - São Paulo, SP - Brasil.

* Secretaria de Saúde do Estado de São Paulo - Rua são Luiz, 99 - São Paulo, SP Brasil. 
GIRON, S. G. et al. - A importância do diagnóstico em nível local para o planejamento de serviço de saúde materna. Rev. Saúde públ., S. Paulo, 8: 43-9, 1974.

natal recebida pela mãe durante a gestação.

Colocando-se a falta de assistência prénatal como um dos fatores responsáveis por óbitos de crianças até os quatro anos, é fácil observar-se que essa assistência supõe um trabalho de elevado teor educativo. Esse trabalho determinaria a diminuição do número de partos domiciliares e conseqüentemente reduziria a atenção não qualificada ao parto; responderia também pela diminuição da infecção puerperal e do tétano neonatorum.

A caracterização de uma área que conte com uma unidade sanitária que não presta serviços em saúde materna leva a várias suposições. Dentre elas, a primeira talvez fosse o pequeno número de gestantes que receberia atenção pré-natal. Outras seriam o número elevado de natimortos e a grande incidência de casos cirúrgicos dentre os partos hospitalares.

Úma área do município de Osasco, no Estado de São Paulo, com uma população geral de 40.134 habitantes $^{1 *}$, possue uma unidade sanitária que não conta com serviço de saúde materna. Este trabalho foi realizado com a finalidade de verificar se uma área com estas características, na realidade, necessita de serviços oficiais de saúde materna, pois, é sabido que em Saúde Pública é difícil aplicar-se um planejamento efetuado para determinada área em outra. Há o consenso geral da importância dele estar baseado em um diagnóstico prévio dos problemas de saúde da área.

\section{MATERIAL E METTODOS}

Considerou-se como universo de estudo uma população de gestantes da área de Quitaúna e Jardim das Flores no Município de Osasco, no período de $1 / 1 / 71$ a $31 / 12 / 71$, que seria determinada por meio de uma amostragem casual simples sem reposição.

A população distribuiu-se de maneira bastante homogênea pelos 286 quarteirões da área delimitada. fazendo que uma amostra de $20 \%$ fosse considerada significativa (57 quarteirões).

As informações referentes às gestações foram obtidas por meio de um formulário preenchido por pessoal treinado **. Foram aplicados 1.038 formulários, no período de $1 / 9 / 72$ a $30 / 9 / 72$.

Todas as tabelas que se referem à apresentação dos dados analisados tiveram seu título simplificado no sentido de subentender tratar-se de "fatos ocorridos na área de Quitaúna e Jardim das Flores, no Município de Osasco, no período de $1 / 1 / 71$ a $31 / 12 / 71 "$.

\section{RESULTADOS E DISCUSSAO}

A população referente à amostra foi de 5.798 habitantes e o número de gestaçóes foi de 215 , sendo 15 delas abortos. Temos, então, um coeficiente geral de natalidade para a área, de $34,49 \%$.

A vitalidade dos recém-nascidos e o número de abortos aparecem na Tabela 1.

TA B E A 1

Vitalidade dos recém-nascidos e número de abortos

\begin{tabular}{l|r|c}
\hline $\begin{array}{l}\text { Vitalidade do } \\
\text { recém-nascido }\end{array}$ & n.o & $\%$ \\
\hline nascidos vivos & $195 \%$ & 90,70 \\
nascidos mortos & 5 & 2,32 \\
abortos & 15 & 6,98 \\
& & \\
\hline Total & 215 & 100,00 \\
\hline
\end{tabular}

* Houve 2 partos gemelares entre os nascidos vivos.

* Esse dado foi corrigido pela pesquísa de campo, dando uma população para a área iguat a 28.990 habitantes.

* Os entrevistadores eram enfermeiras que frequentavam o curso de graduação em Enfermagem de Saúde Pública. 
GIRON, S. G. et al. - A importância do diagnóstico em nivel local para o planejamento de serviço de saúde materna. Rev. Saúde públ., S. Paulo, 8: 43-9, 1974.

Foi possível verificar que a maioria dos partos foram hospitalares e que destes resultaram $97,35 \%$ de nascidos vivos, ao passo que dos 11 ocorridos no domićlio $100 \%$ nasceram vivos (Tabela 2). Esses dados mostraram uma associação perfeita negativa entre o nascimento vivo e o parto hospitalar. A explicação para isso talvez seja o fato que as 5 gestantes que deram a luz a um natimorto tenham apresentado durante a gravidez algum processo patológico que justificou a procura do hospital para o parto, tanto assim que destas pacientes todas tiveram 5 ou mais consultas médicas no periodo prénatal, sendo que duas delas tiveram mais de 11.

TABEL 2

Nascimentos segundo local de ocorrencia

\begin{tabular}{|c|c|c|c|c|}
\hline \multirow{2}{*}{ Nascimentos } & \multicolumn{2}{|c|}{ Hosptial } & \multicolumn{2}{|c|}{ Domicilio } \\
\hline & n. 0 & $\%$ & n. & $\%$ \\
\hline naseidos vivos & 184 & 97,35 & 11 & 100,00 \\
\hline nascidos mortos & 5 & 2,65 & 一 & $一$ \\
\hline Total & 189 & 100,00 & 11 & 100,00 \\
\hline
\end{tabular}

A grande quantidade de partos hospitalares $(94,5 \%)$ supõe que o hospital não foi procurado apenas para os casos complicados, pois $73,80 \%$ dos partos hospitalares foram normais (Tabela 3). Observou-se, também, que os partos domiciliares foram atendidos, talvez em sua totalidade, por pessoa não habilitada (Tabela 4) pois a maioria das mulheres entrevistadas não era capaz de fazer cor-

\section{T A BELA 3}

Distribuição dos partos hospitalares segundo o tipo de parto

\begin{tabular}{l|r|r}
\hline Tipo & n.0 & $\%$ \\
\hline Normal & 138 & 73,80 \\
Cesárea & 43 & 22,99 \\
Forceps & 6 & 3,21 \\
& & \\
\hline Total & 187 & 100,00 \\
\hline
\end{tabular}

TABELA 4

Distribuição dos partos domiciliares segundo a categoria do assistente

\begin{tabular}{l|c|c}
\hline Assistente & n.o & $\%$ \\
\hline Médico & - & $\begin{array}{c}\text { \% } \\
\text { Parteira }\end{array}$ \\
Curiosa & 5 & $\begin{array}{c}45,45 \\
\mathbf{5 4 , 5 5}\end{array}$ \\
\hline Total & 11 & $\mathbf{1 0 0 , 0 0}$ \\
\hline
\end{tabular}

reta distinção entre a parteira habilitada e a curiosa, e não houve parto domiciliar atendido por médico.

Com respeito ao atendimento pré-natal, $96,47 \%$ das gestantes que o procuraram tiveram partos hospitalares, porém, $82,14 \%$ das gestantes que não procuraram esse atendimento também tiveram partos hospitalares (Tabela 5). Essa elevada porcentagem de gestantes sem pré-natal e com parto hospitalar não anulou a exis- 
GIRON, S. G. et al. - A importáncia do diagnóstico em nível local para o planejamento de serviço de saúde materna. Rev. Saúde públ., S. Paulo, 8: 43-9, 1974.

TABELA 5

Local do parto segundo freqüência ao pré-natal

\begin{tabular}{lc|c|c|c|c|c}
\hline $\begin{array}{r}\text { Frequéncla ao } \\
\text { pré-natal }\end{array}$ & Sim & $\%$ & Nåo & $\%$ & Total \\
\hline Local do parto & 164 & 96,47 & 23 & 82,14 & 187 \\
Hospital & 6 & 3,53 & 5 & 17,86 & 11 \\
\hline Domiclilio & 170 & 100,00 & 28 & 100,00 & 198 \\
\hline
\end{tabular}

TABELA 6

Distribuçião das consultas de pré-natal por gestante

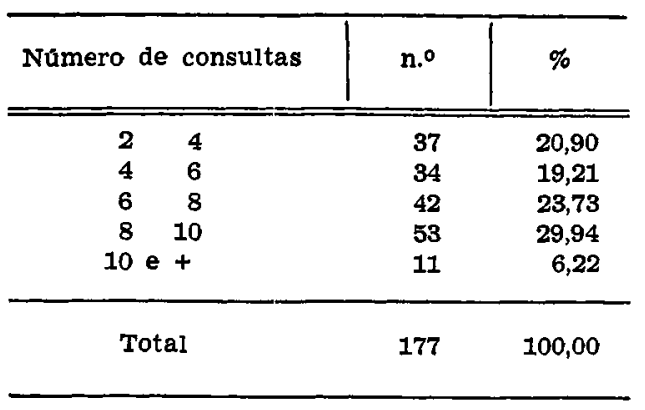

tência de uma associação positiva significante ao nível de $1 \%$ entre a freqüência ao pré-natal e parto hospitalar. Deve-se considerar que $82,86 \%$ das gestantes da área freqüentavam o pré-natal, recebendo quantitativamente atendimento sempre igual ou maior que duas consultas médicas por gestação *. (Tabela 6)

Comparando-se os resultados das gestações entre gestantes com e sem pré-natal, obteve-se o seguinte quadro: os abortamentos apareceram em número maior entre as que não procuraram o atendimento pré-natal; por outro lado, os natimor-

T A B EL A 7

Distribuição do resultado das gestaçōes segundo freqüuência ao pré-natal

\begin{tabular}{|c|c|c|c|c|c|}
\hline $\begin{array}{r}\text { Frequêencia ao } \\
\text { pré-natal }\end{array}$ & Sim & $\%$ & Não & $\%$ & Total \\
\hline $\begin{array}{l}\text { Nascidos vivos } \\
\text { Nascidos mortos } \\
\text { Abortos }\end{array}$ & $\begin{array}{r}167 \\
5 \\
7\end{array}$ & $\begin{array}{r}85,64 \\
100,00 \\
46,60\end{array}$ & $\frac{28}{8}$ & $\frac{14,35}{53,34}$ & $\begin{array}{r}195 \\
5 \\
15\end{array}$ \\
\hline Total & 179 & 83,23 & 36 & 16,75 & 215 \\
\hline
\end{tabular}

- A mediana fol de 6,27 consultas por gestacão. 
GIRON, S. G. et al. - A importância do diagnóstico em nivel local para o planejamento de servico de saúde materna. Rev. Saúde públ., S. Paulo, 8: 43-9, 1974.

tos apareceram apenas entre as que o procuraram (Tabela 7). Observando-se a porcentagem de mulheres que freqüientaram o pré-natal para os diferentes modos pelos quais ocorreu o término das gestações, verificou-se que a menor delas se refere aos abortos $(46,66 \%)$ e os dois grupos apresentam porcentagens mais elevadas, nascidos vivos $75,37 \%$ e nascidos mortos $100 \%$ ). Procurando a causa da associação significante entre a freqüência ao pré-natal e o modo como terminou a gestação, foram construídas tabelas com as proporções mais próximas e testada a mesma hipótese (associação entre freqüência ao pré-natal e modo como ocorreu o término da gestação, com $\alpha=0,01$ ). Como houvesse associação entre os termos não foi possivel determinar estatisticamente a causa da associação.

Entre as gestantes que procuraram o pré-natal apenas $20,11 \%$ receberam vacina anti-tetânica e dessas $62,86 \%$, as 3 doses; no entanto apenas $12,64 \%$ de gestantes receberam dosagens completas. Das gestantes com pré-natal, $9,64 \%$ apresentaram infecção puerperal (Tabela 8). Analisando-se essa tabela verificou-se haver independência entre a presença de infecçōes puerperal e a frequiência ao prénatal a um nível de significância de $1 \%$.

TABELA 8

Distribuição dos casos de infecção puerperal segundo frequência ao pré-natal

\begin{tabular}{|c|c|c|c|c|c|}
\hline Fre & $\operatorname{sim}$ & $\%$ & Não & $\%$ & Total \\
\hline Sim & 16 & 66,66 & 8 & $\mathbf{3 3}, \mathbf{3 4}$ & 24 \\
\hline Não & 161 & 85,18 & 28 & 14,82 & 189 \\
\hline Total & 177 & 83,09 & 36 & 16,91 & 213 \\
\hline
\end{tabular}

TABELA 9

Distribuição das gestantes segundo local de atendimento pré-natal

\begin{tabular}{l|r|c}
\hline Local & $\mathrm{n.0}$ & $\%$ \\
\hline \hline Osasco: & & $\%$ \\
$\quad$ Municipal & 11 & 6,21 \\
$\quad$ Estadual & 0 & \\
$\quad$ Outros & 94 & 53,11 \\
Outros municiplos & 69 & 38,98 \\
Năo informaram & 3 & 1,70 \\
\hline Total & 177 & 100,00 \\
\hline
\end{tabular}

Apenas 11 gestantes $(6,21 \%)$ foram atendidas em serviços de pré-natal mantidos pela prefeitura, e nenhuma recebeu atendimento no serviço mantido pelo Estado, naquele município; $38,98 \%$ delas foram atendidas em outros municípios. (Tabela 9)

\section{COMENTARIOS}

De acordo com os resultados observouse que a maioria das gestantes dessa área $(82,86 \%)$ procurou 0 atendimento de saúde no período gestacional. Verificou- 
GIRON, S. G. et al. - A importâncla do diagnóstico em nivel local para o planejamento de serviço de saúde máterna. Rev. Saûde públ., S. Paulo, 8: 43-9, 1974.

se também, quase como regra geral, que as instituições que lhes ofereceram o atendimento não pertenciam a entidades oficiais. Isso se explica pela existência na área de grande número de indústrias que possuem convênio com sociedades de medicina de grupo para a assistência médica de seus funcionários, sendo a maioria residente na área estudada. A meta prevista pelo plano de saúde para cobertura da população gestante do município de Osasco, naquele período, era de $80 \%^{3}$. A pesquisa evidenciou que na realidade mais de $80 \%$ já estava sendo coberta por este tipo de assistência naquela área. Por outro lado, o número de consultas por gestação teve como mediana o valor de 6,27 o que não só é bastante satisfatório como também excede em 4,27 consultas a meta proposta.

Analisando-se a qualidade do atendimento pelos padróes sugeridos nesse trabalho, apenas seria compreendida a utilidade de uma atenção pré-natal oferecida pelo município àquela área, para cobrir deficiência de vacinação anti-tetânica, pois foram encontrados através de pesquisa realizada nos cartórios de registro civil do município de Osasco e do subdistrito de Jardim América na cidade de São Paulo *, dois casos fatais de tétano neonatorum em crianças nascidas de gestações iniciadas no período pesquisado, procedentes do município de Osasco. Esse número em termos absolutos é pequeno, porém deve ser lembrado que esse tipo de tétano é seguramente evitável através da vacinação materna e que, portanto, não deveria existir. De acordo com Yunes ${ }^{5}$, no período de junho de 1968 a outubro de 1970, foram internados no Hospital das Clínicas da Faculdade de Medicina da Universidade de São Paulo 5 casos de tétano neonatorum procedentes do município de Osasco.
Os outros padrōes sugeridos neste trabalho estavam todos preenchidos: o hospital não foi procurado apenas para casos cirúrgicos, o que fez com que a atenção qualificada ao parto, fosse mínima, dependente apenas do número de partos domiciliares, também pequeno; houve relativamente, poucos nascidos mortos e a presença de infecção puerperal não esteve associada à frequiência ao pré-natal.

Conhecendo-se a falta de recursos humanos e materiais para a Saúde Pública, que grassa pelos paises em desenvolvimento, apenas o não recebimento da vacinação contra o tétano não justifica a manutenção de um serviço de saúde materna pelo municipio, nesta área. Acredita-se que a população orientada quanto a necessidade da vacinação, no período de gestação, irá procurá-la tanto na unidade sanitária como no local onde recebe o atendimento, ficando o problema resolvido apenas com a exigência feita pela Secretaria de Saúde do município às unidades que prestam atendimento pré-natal da vacinação anti-tetânica, e com o oferecimento contínuo pela unidade sanitária de vacina anti-tetânica às gestantes da área.

Verifica-se por esta análise a necessidade de um diagnóstico de saúde até mesmo em nível local, pois um fato que supunha a existência de um problema de saúde pública, quando examinado qualitativamente mostrou sua inexistência, deixando mais uma vez patente a necessidade da análise quantitativa e qualitativa do fato para então constituí-lo ou não em um problema de saúde pública.

\section{CONCLUSOES}

1. A pesquisa evidenciou que a programação para saúde materna necessita

* Esse cartório também foi pesquisado porque os óbitos ocorridos no Hospital das Clinicas da Faculdade de Medicina da USP são al registrados, e a maloria dos casos de tétano ocorridos no municipio de Osasco são encaminhados para esse nosocômio. 
GIRON, S. G. et al. - A importáncia do diagnóstico em nivel local para o planejamento de serviço de saúde materna. Rev. Saúde públ., s. Paulo, 8: 43-9, 1974.

de um diagnóstico da área para identificação das necessidades.

2. A população ao sentir oferta de serviços adequados os procura de forma correta.

3. Os serviços de medicina de grupo necessitam ser assessorados por órgãos oficiais de saúde pública no sentido de aprimorar alguns aspectos importantes da atenção à gestante.
4. Os serviços oficiais de saúde em todos os níveis, ao pretender oferecer assistência na maternidade devem levar em consideração os demais recursos existentes na área a fim de evitar a duplicação de serviços.

5. Recomenda-se também maior entrosamento entre órgãos oficiais e particulares que prestam serviços na área de saúde materna.

RSPU-B/202

GIRoN, S. G. et al. - [The importance of the diagnosis in local level for the program of Maternal Health Services.] Rev. Saúde públ., S. Paulo, 8: $43-9,1974$.

Summary: It was analysed an area in Osasco, São Paulo, with 40,134 inhabitants in order to know how the pregnant women from the area were assisted in this area. This area does not have pre-natal care to assist these pregnant women. A formulary was constructed and applied to a samples of 1,036 families. The results showed a high number of hospital deliveries, analysed $a$ high conantration of prenatal consults (each pregnant woman went to the doctor's 6,27 times) and also showed that the pregnants were assisted by sacieties of groupal medicine. It was concluded that to program Maternal Health Services it is necessary first of all to know the necessity of that service in that area. They recommended that the oficial department of Public Health take in consideration every disposable resource in Maternal Health and give them technical support.

UNITERMS: Maternal Health Services*; Pre-natal care*; Pregnancy*.

\section{REFERENCIAS BIBLIOGRAFICAS}

1. ALONSO, J. - Projeção da população do munictpio de Osasco, São Paulo, Brasil. Osasco, Secretarla de Saúde, Departamento Técnilco-Normativo, 1971.

2. CIARI, C. J. \& ALMEIDA, P. A. M. de - Análise do coeficiente de mortaIldade materna no municiplo de Osasco, São Paulo, Brasil. Rev. Saude públ., S. Paulo, 6: 237-44, 1972

3. OSASCO - Secretaria de Saúde, Departamento Técnico-Normativo - Plano de saude do municipio de Osasco, São Paulo, Brasil. Osasco, 1970. [mimeografado].
4. PUFFER, R. R. \& SERRANO, C. V. Patterns of mortality in childhood. Washington, D.C., Pan American Health Organization, 1973. (Scientific Publications, 262).

5. YUNES, J. - Estudo médico-social do tétano do recém-nascido. São Paulo, 1971. [Tese de doutoramento - Faculdade de Medicina da USP].

Recebido para publicação em 16-12-197s.

Aprovado para publicação em 21-1-1974. 